\title{
CLINICO PATHOLOGICAL REVIEW OF MEGALOBLASTIC ANAEMIA IN CHILDREN- A 7 YEAR PAEDIATRIC HOSPITAL EXPERIENCE
}

M.Ramani1 ${ }^{1}$ D.Ranganath ${ }^{2}$,O. H.RadhikaKrishna ${ }^{3}$, K.Geetha4 ${ }^{4}$, M.Keerthika5, Puja Deshmukh6, S.P.Krupani7, G. Sunitha ${ }^{8}$.

1. Professor, Department of Pathology, Niloufer hospital, Institute of child health, Hyderabad.

2. Professor, Department of Pediatrics, Niloufer hospital, Hyderabad

3. Assistant professor, Department of Pathology, Niloufer hospital, Hyderabad.

4. Assistant professor, Department of Pathology, Niloufer hospital, Hyderabad.

5. III yr , Undergraduate, Osmania Medical College, Hyderabad

6. Post graduate, Department of Pathology, Niloufer hospital, Hyderabad.

7. III yr Undergraduate, Osmania Medical College, Hyderabad

8. Post graduate, Department of Pathology, Niloufer hospital, Hyderabad

\section{CORRESPONDING AUTHOR:}

Dr. M. Ramani,

Niloufer Hospital, Red Hills,

Hyderabad, Andhra Pradesh.

E-mail: drmramani@sify.com

\section{HOW TO CITE THIS ARTICLE:}

M. Ramani, D.Ranganath, O.H. RadhikaKrishna, K.Geetha, M.Keerthika, PujaDeshmukh, S.P.Krupani, G. Sunitha. "Clinico Pathological Review of Megaloblastic Anaemia in Children-A 7 Year Paediatric Hospital Experience."Journal of Evolution of Medical and Dental Sciences 2013; Vol2, Issue 23, June 10; Page: 41364142.

ABSTRACT: INTRODUCTION: Megaloblastic anemia is an anemia that results from inhibition of DNA synthesis. The defect in red cell DNA synthesis is most often due to hypovitaminosis, specifically a deficiency of vitamin $\mathrm{B}_{12}$ or folic acid or both. Megaloblastic anemia has a slow onset, the symptoms develop rather slowly, especially when compared to that of other anemias. It has varied clinical manifestations. AIM: To study various clinical manifestations of megaloblastic anemia in children. To describe the blood smear and bone marrow morphology characteristics of megaloblastic anemia. MATERIALS AND METHODS: A study of 124 cases of megaloblastic anemia in children below 15 years of age during 7 years (January 2006 to December 2012) was conducted at Niloufer hospital. Clinical findings were obtained and statistical analysis of the data was done. RESULTS: More than half of the patients presented with mild anemia and pallor. Other presenting features included fever (14.4\%), splenomegaly (23.4\%) and bleeding manifestations (15.3\%). CONCLUSION: The study concludes that pallor is not the only presenting complaint of megaloblastic anemia. It can have varied clinical presentations like hepato splenomegaly and thrombocytopenia with bleeding manifestations. Prompt diagnosis is especially important as megaloblastic anemia is a completely curable condition.

KEY WORDS: Megaloblastic Anemia, Deficiency of vitamin $B_{12}$, Deficiency of folic acid, Pallor, Inhibition of DNA synthesis, Splenomegaly, Bleeding manifestations. 
INTRODUCTION: Megaloblastic Anemia is one of the important causes of Anemias in children. It results from abnormal maturation of hematopoietic cells due to faulty DNA synthesis. Two vitamins, cyanocobalamin (vitamin B12) and folic acid are essential for DNA biosynthesis. Deficiency of either vitamin results in abnormal nuclear maturation with normal cytoplasmic maturation, apoptosis, ineffective erythropoiesis, intramedullary hemolysis, pancytopenia and typical morphological abnormalities in blood and marrow cells (1). The morphological hallmark of megaloblastic anemia is megaloblastic change of all erythroid precursors [Figure 4]. Megaloblasts are large cells with an increased nuclear / cytoplasmic ratio in which nuclear maturation is delayed, while cytoplasmic maturation is more advanced.

Diagnosing this disease assumes great clinical importance since it responds exceedingly well to treatment.

MATERIALS AND METHODS: We reviewed retrospectively 124 cases of megaloblastic anemia during 7 years, from January 2006 to December 2012, in children below 15 years of age at Niloufer hospital.

The diagnostic approach to megaloblastic anemia involved three steps. The first step was obtaining a complete clinical history which included fever, bleeding manifestations like hematemesis, epistaxis and bleeding gums. Second step being physical examination, where we looked for splenomegaly, petechial hemorrhages. Third step included complete blood picture and bone marrow examination.(2) Based on the blood hemoglobin levels patients were categorized into mild $(10-11 \mathrm{~g} / \mathrm{dl})$, moderate $(7-10 \mathrm{~g} / \mathrm{dl})$ and severe anemia $(<7 \mathrm{~g} / \mathrm{dl})$.

RESULTS: Out of the 124 cases studied in the period of 7 years, anemia was found to be more common in the age group of 11-15 years. Incidence of megaloblastic anemia was slightly higher in boys, 63 cases (50.8\%) compared to girls, 61 cases (49.2\%). About half of the patients (44.35\%) presented with mild anemia [Table 1]. These patients mainly presented with dyspnea, fatigue, palpitations. One fourth cases (23.4\%) presented with mild splenomegaly.

Cytopenias are a common manifestation of megaloblastic anemia. Most of the patients, 48 cases(38.7\%) presented with only anemia. The peripheral smear of the majority of cases showed normocytic normochromic red cells, macrocytes, macrovalocytes (Figure 1) and hypersegmented polymorphs. 42 cases (33.9\%) presented with a combination of anemia and thrombocytopenia. The bone marrow was predominantly hyper cellular with megaloblastoid changes in the erythroid series, large band forms and metamyelocytes (Figure 2,3). Suppression of all the bone marrow precursors leading to anemia, thrombocytopenia and leucopenia was seen in 34 cases $(27.4 \%)$ [Table 2].

Bleeding was mainly into the skin and subcutaneous tissue while five children also had epistaxis. 19 cases (15.3\%) presented with bleeding and severe anemia making them clinically indistinguishable from aplastic anemia. Hemorrhagic emergencies namely intracranial bleeding and gut bleeding occurred in one case each.

Twenty-four cases (19.4\%) presented with severe anemia, bleeding, hepatosplenomegaly and fever mimicking acute leukemia [Table 3]. Eleven cases were provisionally diagnosed as chronic malaria as they presented with anemia, fever and 
splenomegaly Cases which were suspected to be acute leukemia, aplastic anemia, chronic malaria, turned out to be megaloblastic anemia, which were further evaluated by clinicians by vitamin assays to pinpoint the deficiency to correctly treat the anemia.

DISCUSSION: Megaloblastic anemia is an anemia that results from inhibition of DNA synthesis in red blood cell production. When DNA synthesis is impaired, the cell cycle cannot progress from the G2 growth stage to the mitosis (M) stage. This leads to continuing cell growth without division, which causes nuclear-cytoplasmic asynchrony and presents as macrocytosis (3) [Figure 1]. Megaloblastic anemia has a slow onset, the symptoms develop rather slowly, especially when compared to that of other anemias. The defect in red cell DNA synthesis is most often due to hypovitaminosis, specifically a deficiency of vitamin $\mathrm{B}_{12}$ or folic acid or both ${ }^{(4)}$ [Table 4]. Vitamin $B_{12}$ deficiency alone will not cause the syndrome in the presence of sufficient folate, for the mechanism is loss of $\mathrm{B}_{12}$ dependent folate recycling, followed by folate-deficiency which results in loss of nucleic acid synthesis leading to defects in DNA synthesis. Folic acid supplementation [Table 5] in the absence of vitamin $\mathrm{B}_{12}$ prevents this type of anemia, but the neurological manifestations of vitamin $\mathrm{B}_{12}$ deficiency cannot be prevented. Loss of micronutrients may also be a cause.

Megaloblastosis is characterized by many large immature and dysfunctional red blood cells (megaloblasts) in the bone marrow [Figure 4] and also by hypersegmented neutrophils having five or more nuclear lobes in more than $5 \%$ of cells in the peripheral smears

In a study done by Vineetha et al with a total of 106 cases, most common presenting feature was generalized weakness, followed by pallor and fever which was 83.49 $\%$ and $53.49 \%$ respectively. Splenomegaly was seen in 19 cases. Whereas in our study, among 124 cases, about half of the patients (44.35\%) presented with mild anemia [ Figure 5]. These patients mainly presented with dyspnea, fatigue, palpitations. One fourth cases $(23.4 \%)$ presented with mild splenomegaly. We have also taken bleeding into consideration and it was seen that nineteen cases (15.3\%) presented with bleeding and severe anemia making them clinically indistinguishable from aplastic anemia. Hemorrhagic emergencies namely intracranial bleeding and gut bleeding occurred in one case each. Thus, we could successfully rule out possibility of malignancy in twenty-four cases(19.4\%) which presented with severe anemia, bleeding, hepatosplenomegaly and fever actually mimicking acute leukemia [Table 3].

In a study conducted by Gayatri et al with 104 cases, the commonest physical finding was pallor, followed by splenomegaly, generalized weakness and fever. Pancytopenia was present in $62 \%$ of patients. Dimorphic anemia was predominant unlike in our study. Bone marrow aspiration was done in all the cases to rule out aplastic anemia. It was suggestive that presence of pallor and splenomegaly are not the only indications of megaloblastic anemia, a complete clinical history, involving any history of fever, bleeding manifestations like hematemesis and epistaxis, physical examination, complete blood picture and bone marrow examination should be performed to rule out all the causes of megaloblastic anemia and other possibilities of pallor, splenomegaly. This study also suggests that anemia must be an important differential diagnosis in patients presenting with pancytopenia(7).

Megaloblastic anemia is a chronic condition developing over a period of time and most patients are well compensated. There is no indication for urgent blood transfusion. 
Serum samples for assay of the 2 vitamins (B12 and folic acid) should be drawn before any form of therapy is given since assays alone can determine which vitamin is deficient(2).

The average Indian vegetarian diet is deficient in cobalamin. It is possible that the increased demand during growth spurt, puberty and child-bearing age group in a population already deficient in cobalamin precipitates the anemia.

The lining epithelium of the gastrointestinal tract becomes atrophic in megaloblastosis. A vicious cycle of megaloblastosis leading to atrophy of mucosa, and subsequent malabsorption of the two vitamins, worsens megaloblastic anemia(5).

For a laboratory diagnosis of megaloblastic anemia (6), a Complete Blood Picture with red cell indices, examination of a well stained blood film and assay of the 2 vitamins are sufficient to make a definitive diagnosis.

In conclusion, megaloblastic anemia causes substantial morbidity in patients with anaemia. Megaloblastic anaemia must be considered in the differential diagnosis of patients presenting with pyrexia of unknown origin, mild icterus or pancytopenia.

A large volume of recent literature links serum levels of homocysteine and methyl malonic acid in cobalamin and folate deficiency to occlusive cardiovascular disease and neurological manifestations ${ }^{(8)}$. Complete evaluation for subtle neurological signs and cardiac function needs to be done in the at-risk population to assess the deficiency of these vitamins ${ }^{(1)}$.

CONCLUSION :Megaloblastic anemia patients do not necessarily always present with only pallor. On the basis of observations made in this study, it is clear that megaloblastic anemia can present with varied and confusing clinical manifestations. Strong suspicion of megaloblastic anemia should always be entertained by the clinicians to improve clinical outcome. Bone marrow examination always proves to be diagnostic and conclusive.

\section{REFERENCES}

1. Uma khanduri, Archna sharma, Megaloblastic anaemia: Prevalence and causative factors, National Medical Journal of India, 2007; 20(4), 172-175.

2. Ralph Caramel, MD, Laboratory diagnosis of megaloblastic anemia, Western Journal of Medicine, 1978; 128(4), 294-394.

3. Florence Asliria, MD, Megaloblastic anemia and other causes of macrocytosis, Clinical medicine and research, 2006; 4(3); 236-241.

4. Vineetha Unnikishnan et al, Clinico-aetiologic profile of macrocytic anemias with special reference to megaloblastic anemia, Indian Journal of Hematology and Blood transfusions, 2008; 24(4), 155-165.

5. James A Halsted, Megaloblastic anemia associated with surgically produced gastro intestinal abnormalities, Western Journal of Medicine; 1955; 83(3), 212-217.

6. Ralph 0 Wallerstein, Jr MD, Laboratory evaluation of anemia, Western Journal of Medicine, 1987; 146(4), 443-451.

7. B.N. Gayatri, Kadam Satyanarayan Rao, Pancytopenia: A Clinico-hematological study, Journal of laboratory physicians, 2011; 3(1), 15-20.

8. Elvira M. Guerra Shinohara et al, Elevated serum S-Adenosyl homocysteine in cobalamin deficient megaloblastic anemia, Metabolism, 2007; 56(3), 339-347. 


\section{ORIGINAL ARTICLE}

Table 1: Hemoglobin levels in patients suffering from megaloblastic anemia, with respect to gender

\begin{tabular}{|l|l|l|l|}
\hline Level(gm/dl) & Boys & Girls & Total \\
\hline $10-11($ mild anemia) & $30(24.19 \%)$ & $25(20.16 \%)$ & $55(44.35 \%)$ \\
\hline $7-10$ (moderate anemia) & $15(12.1 \%)$ & $19(15.32 \%)$ & $34(27.42 \%)$ \\
\hline$<7($ severe anemia) & $18(14.51 \%)$ & $17(13.7 \%)$ & $35(28.2 \%)$ \\
\hline Total & $63(50.8 \%)$ & $61(49.2 \%)$ & $124(100 \%)$ \\
\hline
\end{tabular}

Table 2: Prevalence of Cytopenias in Megaloblastic Anemia

\begin{tabular}{|l|l|}
\hline Cytopenias & Cases \\
\hline Anemia & $48(38.7 \%)$ \\
\hline Anemia+ Thrombocytopenia & $42(33.9 \%)$ \\
\hline Anemia+ Thrombocytopenia+ Leucopenia & $34(27.4 \%)$ \\
\hline Total & $124(100 \%)$ \\
\hline
\end{tabular}

Table 3: Spectrum of clinical presentation in Megaloblastic Anemia

\begin{tabular}{|l|l|l|l|}
\hline Symptoms & Boys & Girls & Total \\
\hline Anemia (A) & $20(16.1 \%)$ & $14(11.3 \%)$ & $34(27.4 \%)$ \\
\hline A + Splenomegaly & $12(9.7 \%)$ & $17(13.7 \%)$ & $29(23.4 \%)$ \\
\hline A + Bleeding manifestations & $11(8.9 \%)$ & $8(6.4 \%)$ & $19(15.3 \%)$ \\
\hline $\begin{array}{l}\text { A + Splenomegaly, Bleeding } \\
\text { manifestations, Fever }\end{array}$ & $14(11.3 \%)$ & $10(8.1 \%)$ & $24(19.4 \%)$ \\
\hline A + Fever & $6(4.8 \%)$ & $12(9.6 \%)$ & $18(14.4 \%)$ \\
\hline Total & $63(50.8 \%)$ & $61(49.2 \%)$ & $124(100 \%)$ \\
\hline
\end{tabular}

Table 4: Recommended dietary allowances for Vitamin B12

\begin{tabular}{|l|l|}
\hline Age & Boys and Girls( g/day) \\
\hline $0-6$ months & 0.4 \\
\hline 7-12 months & 0.5 \\
\hline 1-3 years & 0.9 \\
\hline 4-8 years & 1.2 \\
\hline 9-13 years & 1.8 \\
\hline$>14$ years & 2.4 \\
\hline
\end{tabular}




\section{ORIGINAL ARTICLE}

Table 5: Recommended dietary allowances for folate:

\begin{tabular}{|l|l|}
\hline Age (years) & Boys and girls(g/day) \\
\hline $0-3$ & 150 \\
\hline $4-8$ & 200 \\
\hline $9-13$ & 300 \\
\hline $14-18$ & 400 \\
\hline $19+$ & 400 \\
\hline
\end{tabular}

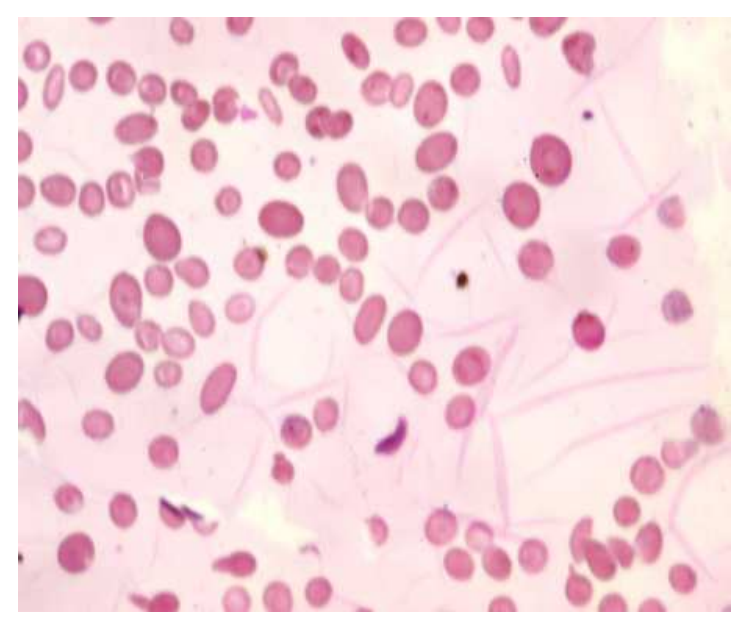

Figure 1: Peripheral blood smear showing anisocytosis of R.B.C [Leishman's staining, 40x magnification]

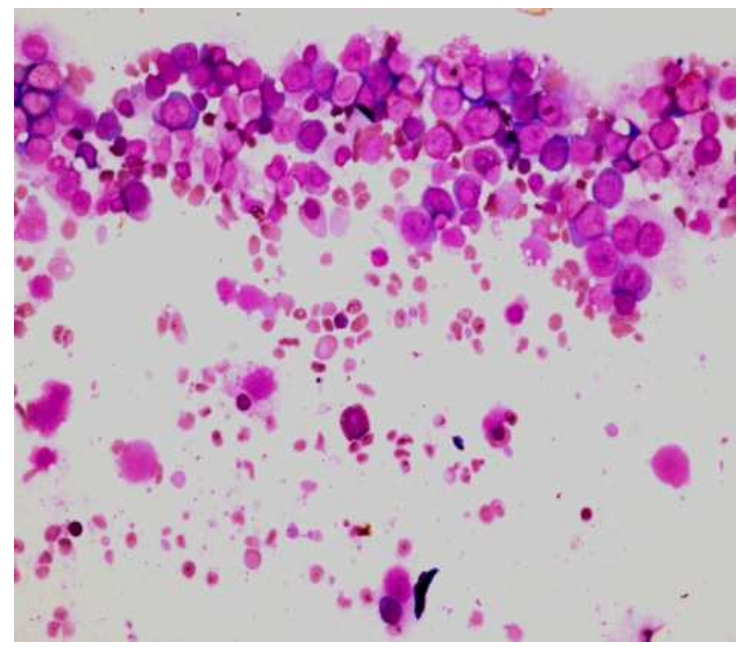

Figure 2: Bone marrow aspiration showing erythroid hyperplasia [Leishman's staining, 40x magnification] 


\section{ORIGINAL ARTICLE}

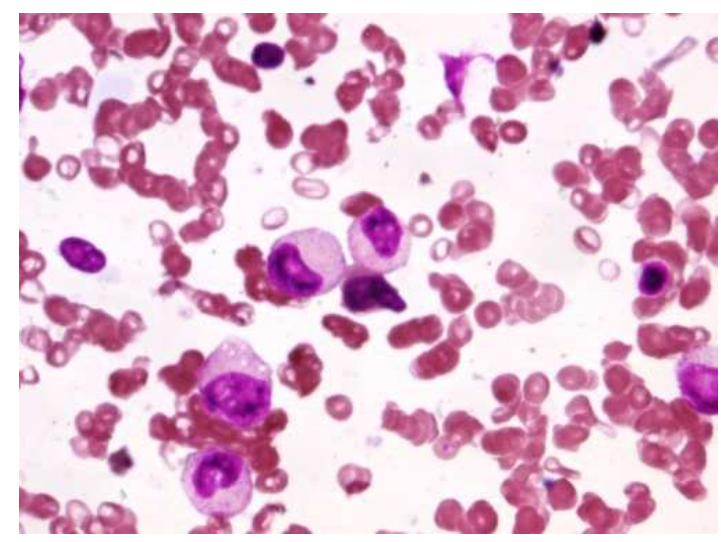

Figure 3: Bone marrow aspiration showing a large band form [Leishman's staining, 100x magnification

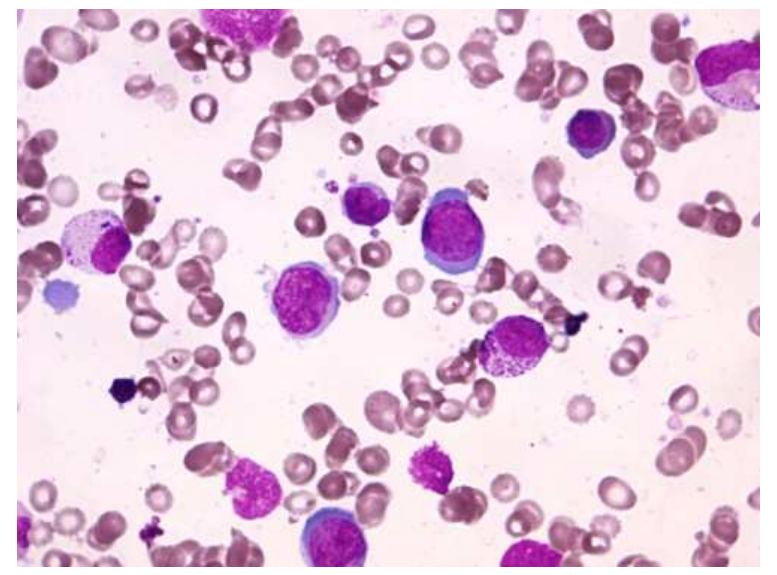

Figure 4: Bone marrow aspiration showing megaloblasts (with open sieve like chromatin) Leishman's stain, 100x

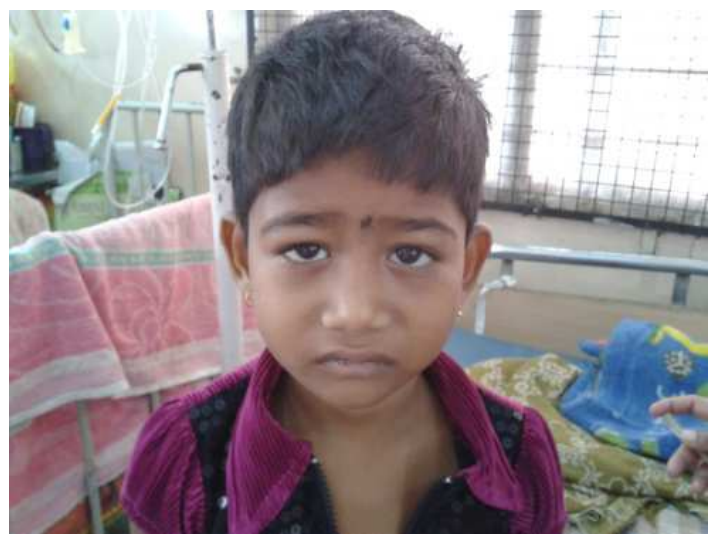

Figure 5: A 6 year old girl suffering from megaloblastic anemia presented with fatigue, lack of energy $\&$ anemia 